\title{
Analysis of Electric Propulsion System for Exploration of Saturn
}

\author{
Carlos Renato Huaura Solórzano, ${ }^{1}$ Antonio Fernando Bertachini \\ de Almeida Prado, ${ }^{1}$ and Alexander Alexandrovich Sukhanov ${ }^{2}$ \\ ${ }^{1}$ Division of Space Mechanics and Control, National Institute for Space Research (INPE), \\ 12227010 São José dos Campos, Brazil \\ ${ }^{2}$ Flight Dynamics and Data Processing Division, Space Research Institute (IKI), 117997 Moscow, Russia
}

Correspondence should be addressed to Carlos Renato Huaura Solórzano, renato@dem.inpe.br

Received 3 November 2008; Revised 11 May 2009; Accepted 24 June 2009

Recommended by Dane Quinn

Exploration of the outer planets has experienced new interest with the launch of the Cassini and the New Horizons Missions. At the present time, new technologies are under study for the better use of electric propulsion system in deep space missions. In the present paper, the method of the transporting trajectory is used to study this problem. This approximated method for the flight optimization with power-limited low thrust is based on the linearization of the motion of a spacecraft near a keplerian orbit that is close to the transfer trajectory. With the goal of maximizing the mass to be delivered in Saturn, several transfers were studied using nuclear, radioisotopic and solar electric propulsion systems.

Copyright (C) 2009 Carlos Renato Huaura Solórzano et al. This is an open access article distributed under the Creative Commons Attribution License, which permits unrestricted use, distribution, and reproduction in any medium, provided the original work is properly cited.

\section{Introduction}

The first mission to Saturn was Pioneer 11 that was launched on April 5, 1973. After making a flyby in Jupiter it determined the mass of the Jupiter's moon Callisto. Looping high above the ecliptic plane and across the Solar System, Pioneer 11 raced toward its appointment with Saturn on September 1, 1979. Voyager 2 was launched on August 20, 1977, and Voyager 1 was launched on September 5, 1977. Voyager 1 reached Saturn on November 12, 1980, followed by Voyager 2 in August 25, 1981. Later, the Cassini-Huygens spacecraft was launched on October 15, 1997. Using the gravity assist technique with the combination Venus-Venus-Earth and Jupiter the spacecraft increased its velocity to a level high enough to reach its final destination. On July 1, 2004, the Cassini-Huygens spacecraft fired its main engine to reduce its speed, allowing the spacecraft to be captured by Saturn's gravity and enter orbit. In May 28, 2008, the Cassini spacecraft passed by Saturn's moon Titan and made its last flyby of the original four-year tour, but Cassini's exploration of Saturn will continue for two more years. 
With the advent of both the Deep Space 1 mission (Brophy and Noca [1]) and the geosynchronous orbit insertion of a spacecraft, Electric Propulsion (EP) has moved from providing station-keeping capability for spacecrafts to be able to act as its primary propulsion system. More payload mass can be delivered than would have been possible with chemical propulsion due to the propellant reduction achieved. This reduction is due to the specific characteristics of EP systems: lowthrust, high efficiency, and long-burntimes.

\section{General Information}

The performance of a one-stage propulsion system can be roughly characterized by two variables: the maximum ejection velocity, which can be related with the maximum specific impulse and the ratio between the maximum thrust and the engine weight on the ground.

Concerning these two parameters (Marec [2]), the propulsion system can be classified into high-thrust system (HT), characterized by a high-thrust acceleration level and a low specific impulse (conventional propulsion), and low-thrust system (LT) characterized by a low-thrust acceleration level and a high specific impulse (electric propulsion).

Concerning the operating domain, the propulsion system can be essentially classified into constant ejection velocity (CEV) limited thrust systems, and limited power (LP) variable ejection velocity systems.

For the case of the idealized LP system, the only constraint concerns to the power $\left(W \leq W_{\max }\right)$. More details are available in Marec [2].

In this paper we studied the electric propulsion system of low-thrust type with limited power. Besides, the low-thrust transfers that are studied here have no constraint on the thrust direction. In general, these constraints can be caused by peculiarities of the attitude control system and the mode of the stabilization of the spacecraft.

\section{Mathematical Model for the Optimization of the Low Thrust Transfers}

A description of the mathematical models used to study the low-thrust transfers is now made, in order to explain the procedures used in the present paper.

\subsection{Optimization of the Low Thrust}

The electric propulsion low-thrust system uses the ionization of a propellant and its subsequent acceleration in an electrostatic field or electromagnetic to generate thrust. In systems with chemical propulsion, the ignition of the engine can run for several minutes, but in the case of the systems that use the electric propulsion, the ignition of the engine needs to run for longer times, up to several months in some cases.

The equations of motion are

$$
\dot{\vec{r}}=\vec{V}, \quad \dot{\vec{V}}=\vec{f}_{v}+\vec{\alpha} .
$$

In this way, the general equations of motion for trajectories obtained by the use of the lowthrust propulsion system are the equations of motion of the problem of two bodies with the 
inclusion of an additional term that represents the acceleration due to the propulsive force. The cost function (i.e., the functional to be minimized) is

$$
J=x^{0}\left(t_{1}\right)=\int_{t_{0}}^{t_{f}} f^{0}\left(\vec{r}, \vec{f}_{v}, \vec{\alpha}\right) d t
$$

The Hamiltonian is

$$
H=p_{0} f^{0}+\vec{p}_{r}^{T} \vec{v}+\vec{p}_{v}^{T} \vec{f}_{v}+\vec{p}_{v}^{T} \vec{\alpha}
$$

If $\vec{f}_{v}=\left\{f^{0}, \vec{v}, \vec{f}_{v}, \vec{\alpha}\right\}$ does not depend on $x^{0}=x^{0}(t)$ then

$$
H=-f^{0}+\vec{p}_{r}^{T} \vec{v}+\vec{p}_{v}^{T} \vec{f}_{v}+\vec{p}_{v}^{T} \vec{\alpha}
$$

The effective power is

$$
W_{e}=\eta W=\frac{\dot{m}_{p} u^{2}}{2}
$$

Usually $W_{e}, u$ are given by

$$
\begin{gathered}
\dot{m}_{p}=-\dot{m}=\frac{2 W_{e}}{u^{2}}, \\
F_{T}=\dot{m}_{p} u=\frac{2 W_{e}}{u}, \\
\alpha=\frac{F_{T}}{m}=\frac{2 W_{e}}{m u} .
\end{gathered}
$$

The limited power problem ((LP) problem) has only an upper limit of the power given, that is $0 \leq W_{e} \leq W_{e m}$. the exhaust velocity can be varied inside some limits, that is, $u_{\min }<u<$ $u_{\max }$. The acceleration can be arbitrarily varied in the interval $\alpha_{\min } \leq \alpha<\alpha_{\max }$. Equations (3.5)-(8) show that

$$
\dot{m}_{p}=\frac{m^{2} \alpha^{2}}{2 W_{e}} \geq \frac{m^{2} \alpha^{2}}{2 W_{e m}}
$$

In this way, the maximum power provides a minimum propellant consumption. For LP problems $\left(\mathrm{u}_{\min }<u<\mathrm{u}_{\max }\right)$, due to (3.7) and the relation $\dot{m}=-\dot{m}_{p}$

$$
\frac{\alpha^{2}}{2 W_{e}}=\frac{\dot{m}}{m^{2}}=\frac{d}{d t}\left(\frac{1}{m}\right) \longrightarrow \frac{1}{2} \int_{t_{0}}^{t_{f}} \frac{\alpha^{2}}{W_{e}} d t=\frac{1}{m_{1}}-\frac{1}{m_{0}}
$$


the performance index can be taken as

$$
J=\frac{1}{2} \int_{t_{0}}^{t_{f}} \frac{\alpha^{2}}{W_{e}} d t \longrightarrow \min
$$

Recalling (3.4), from (3.9) the Hamiltonian is

$$
\begin{aligned}
H & =-\frac{\alpha^{2}}{W_{e}}+\vec{p}_{r}^{T} \vec{v}+\vec{p}_{v}^{T} \vec{f}_{v}+\vec{p}_{v}^{T} \vec{\alpha} \\
\frac{\partial H}{\partial \vec{\alpha}} & =-\frac{\vec{\alpha}^{T}}{2 W_{e}}+\vec{p}_{v}^{T}=\overrightarrow{0}^{T}
\end{aligned}
$$

where the optimal thrust is

$$
\vec{\alpha}=W_{e} \vec{p}_{v}
$$

For the case of constant power, it can be taken as

$$
\begin{aligned}
J & =\frac{1}{2} \int_{t_{0}}^{t_{f}} \alpha^{2} d t \longrightarrow \text { min }, \\
\vec{\alpha} & =\vec{p}_{v} .
\end{aligned}
$$

For the solar power, due to the variation with the inverse square of the distance from the Sun, we have

$$
\begin{aligned}
& J=\frac{1}{2} \int_{t_{0}}^{t_{f}}(r \alpha)^{2} d t \longrightarrow \min , \\
& \vec{\alpha}=\frac{\vec{p}_{v}}{r^{2}} .
\end{aligned}
$$

For the analysis of the low-thrust trajectories, the transporting trajectory method is used.

\subsection{The Method of the Transporting Trajectory}

This approximated method of optimization of flight with ideally controlled small thrust is based on the linearization of the motion of a spacecraft near some reference Keplerian orbit (transporting trajectory). The equation of motion of the spacecraft is

$$
\begin{aligned}
\dot{\vec{x}} & =\vec{f}(\vec{x})+\vec{g}, \\
\vec{f}(\vec{x}) & =\left\{\vec{v}, \frac{\mu}{r^{3}} \vec{r}\right\}, \quad \vec{g}=\{\overrightarrow{0}, \vec{\alpha}\}
\end{aligned}
$$


with boundary values

$$
\vec{x}\left(t_{0}\right)=\vec{x}_{0}, \quad \vec{x}\left(t_{1}\right)=\vec{x}_{1} .
$$

Now, considering the associated Keplerian motion described by equation

$$
\dot{\vec{x}}=\vec{f}(\vec{x}),
$$

and let $\vec{y}=\vec{y}(t)$ be a solution of (3.17), with given boundary values

$$
\vec{y}\left(t_{0}\right)=\vec{y}_{0}, \quad \vec{y}\left(t_{1}\right)=\vec{y}_{1} .
$$

Note that the positions in the state vectors $\vec{y}_{0}, \vec{y}_{1}$ are given and the corresponding spacecraft velocities can be found by solving a Lambert's problem. The solution of (3.14) with boundary values (3.16) is in the form

$$
\vec{x}=\vec{\xi}+\vec{y}
$$

due to the low-thrust problem, it is reasonable to assume that

$$
\|\vec{\xi}\| \ll\|\vec{y}\|
$$

A linearization of (3.14) for $\vec{\xi}$ yields

$$
\begin{gathered}
\dot{\vec{\xi}}=F \vec{\xi}+\vec{g}, \\
F=\frac{\partial \vec{f}}{\partial \vec{x}}=\left[\begin{array}{ll}
0 & I \\
G & 0
\end{array}\right], \quad G=\frac{\mu}{r^{3}}\left(\frac{3 \vec{r} \vec{r}^{T}}{r^{2}}-I\right) .
\end{gathered}
$$

Boundary values for $\vec{\xi}$ are

$$
\vec{\xi}\left(t_{0}\right)=\vec{x}_{0}-\vec{y}_{0}=\vec{\xi}_{0}, \quad \vec{\xi}\left(t_{1}\right)=\vec{x}_{1}-\vec{y}_{1}=\vec{\xi}_{1}
$$

The Keplerian orbit given by $\vec{y}=\vec{y}(t)$ is called a reference orbit or a transporting trajectory. Matrices (3.22) are calculated in this orbit.

The Hamiltonian for the linearized problem is

$$
H=-\frac{\alpha^{2}}{2 W_{e}}+\vec{p}^{T} F+\vec{p}_{v}^{T} \vec{\alpha},
$$


where

$$
\vec{p}=\left\{\vec{p}_{r}, \vec{p}_{v}\right\}
$$

is the adjoint (costate) variable.

More details of the analysis of the trajectories using low thrust and the transporting trajectory method can be found in Beletsky and Egorov [3], Sukhanov [4, 5].

\section{Results of the Mission Analysis}

A power-limited low-thrust transfer to Saturn is considered in this paper. For all cases considered below it is assumed that the spacecraft leaves the Earth's sphere of influence with variable velocity and approaches Saturn with zero velocity. Several analyses were considered as a function of the ratio between the final and initial mass, time of flight (TOF), velocity at infinity, and effective specific power. The unit of power used in this paper is the effective specific power that is [efficiency* (total power/total mass)]. Besides, for all cases, the efficiency considered is $100 \%$. In this paper, the solar arrays degradation and decay of the nuclear/radioisotope source are not considered. But the nuclear/radioisotope power is considered constant. On the other way, the variation of the solar power is considered, taking into account the distance from the Sun. The solar power of the solar arrays follows the rule of the inverse of the square of the spacecraft heliocentric distance. The planetary state vector obtained from the planetary ephemerides (J2000) is used, together with Chebyshev's interpolation. The tolerance used is $10^{-8}$. After analyzing several dates of launch between 2020 and 2030, we choose the option in May 06, 2021.

\subsection{Nuclear Electric Propulsion}

Nuclear Electric Propulsion (NEP) uses a reactor power system to provide the electricity for thrusters that ionize and accelerate propellant to produce thrust. The application of nuclear systems in space has advantage in situations where the distances from the Sun are large and the solar power density is too low $\gtrsim 1.5 \mathrm{AU}$ ) and locations where solar power is not readily or continuously available. One of the key performances for NEP is the power system specific mass, measured in $\mathrm{kg} / \mathrm{kW}$. Low values are desired in order to provide the maximum mass allocation for payload or propellant. High values result in minimal delivered payloads.

In this section, the study of the NEP system for a trip to Saturn is performed. Figure 1 shows the trajectory of the flight Earth-Saturn projected on the ecliptic plane. Figure 2 shows that the use of NEP allows the delivery of larger masses to the target planet, due to the high power of this system.

When the TOF is larger, the propellant consumption is smaller. These cases showed that the NEP is a good way to transport large payloads to Saturn. Considering the cases with $V_{\text {inf }}=5 \mathrm{~km} / \mathrm{s}$ and time of flight of 5 years, the mass relation is between $0.86 \leq m_{f} / m_{0} \leq 0.955$. Note also that the delivered mass increases significantly with trip time. Increasing the velocity at infinity, the mass ratio also increases. However, the propellant consumption is high when the time of flight is short. 


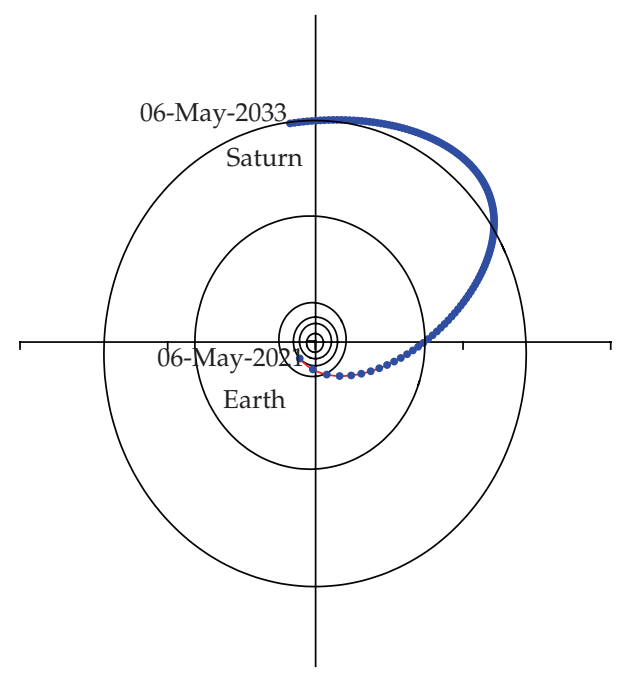

Figure 1: Trajectory of the flight Earth-Saturn with effective specific power $(\mathrm{NEP})$ of $50 \mathrm{~W} / \mathrm{kg}$ and $V_{\text {inf }}=$ $10 \mathrm{~km} / \mathrm{s}$ on Earth's sphere influence (TOF $=12$ years).

The angle of the thrust with the spacecraft velocity is shown in Figure 3(a) (continuous lines), however the angles of the thrust with the orbital plane take small values due to the position of the Earth and Saturn near the ecliptic plane (discontinuous lines).

The behavior of the thrust is presented in Figure 3(b) (continuous lines). It is visible that the thrust has high values at the beginning of the transfers, the specific impulse (discontinuous lines) shows an opposite relative behavior, that is, when the thrust has high values the specific impulse has low values. The propulsion system offers values of variable thrust.

\subsection{Radioisotope Electric Propulsion (REP)}

The Deep Space 1 mission used a radioisotope thermoelectric generator that was combined with off-the-shelf ion propulsion systems. This combination provides a combined specific mass of almost $300 \mathrm{~kg} / \mathrm{kW}$. However, advanced radioisotope power system that is under development could achieve the specific mass of $150 \mathrm{~kg} / \mathrm{kW}$ or lower required for REP. Figure 4 shows the trajectory of the flight Earth-Saturn projected on the ecliptic plane.

In this section, some simulations for the REP system are shown. Figure 5 shows the use of radioisotope power source. When the TOF is larger, the propellant consumption is smaller. These cases showed that the REP is another way to transport payloads to Saturn. For the cases where $V_{\text {inf }}=5 \mathrm{~km} / \mathrm{s}$ and the time of flight is 5 years, the mass ratio are between $0.45 \leq m_{f} / m_{0} \leq 0.67$.

The angle of the thrust with the spacecraft velocity is shown in Figure 6(a) (continuous lines) as well as the angle that the thrust makes with the orbital plane (discontinuous lines).

The behavior of the thrust is presented in Figure 6(b) (continuous lines) and the specific impulse (discontinuous line).

\subsection{Solar Electric Propulsion (SEP)}

In October 1998, NASA launched the Deep Space 1 that was the first interplanetary mission to be propelled by solar electric propulsion (Rayman and Williams [6], Brophy and Noca [1]). 


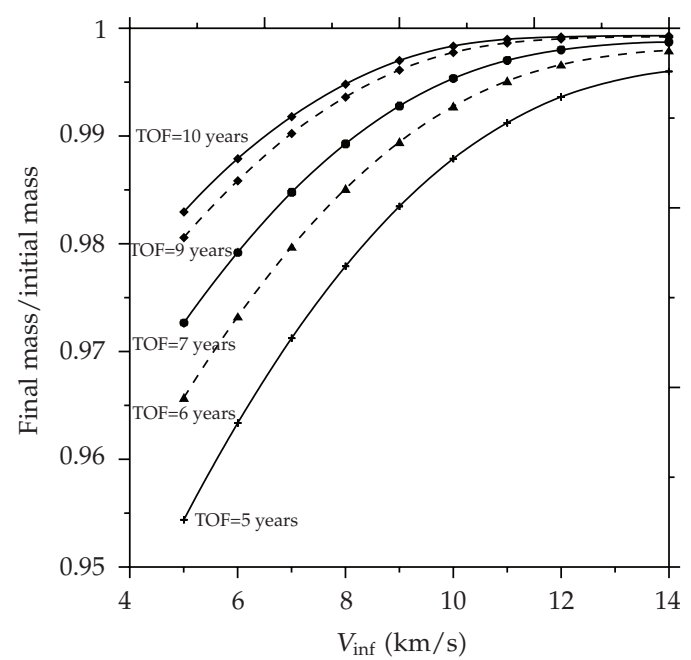

(a)

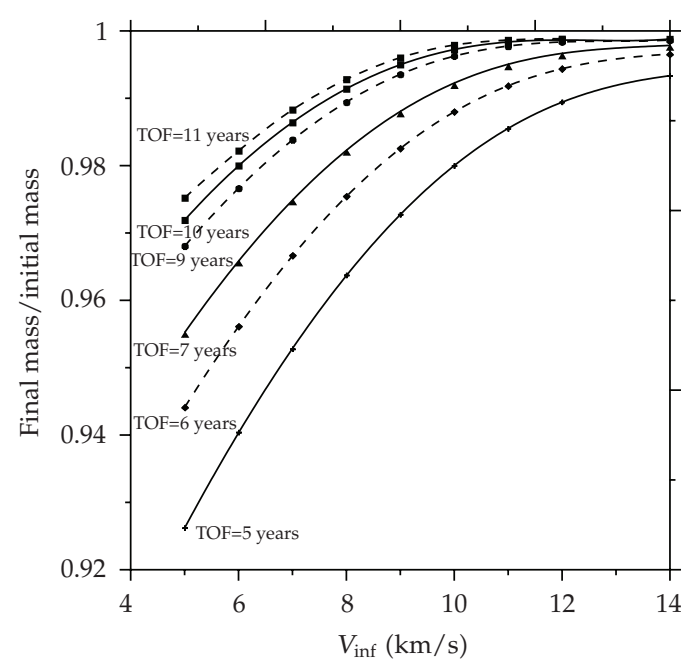

(c)

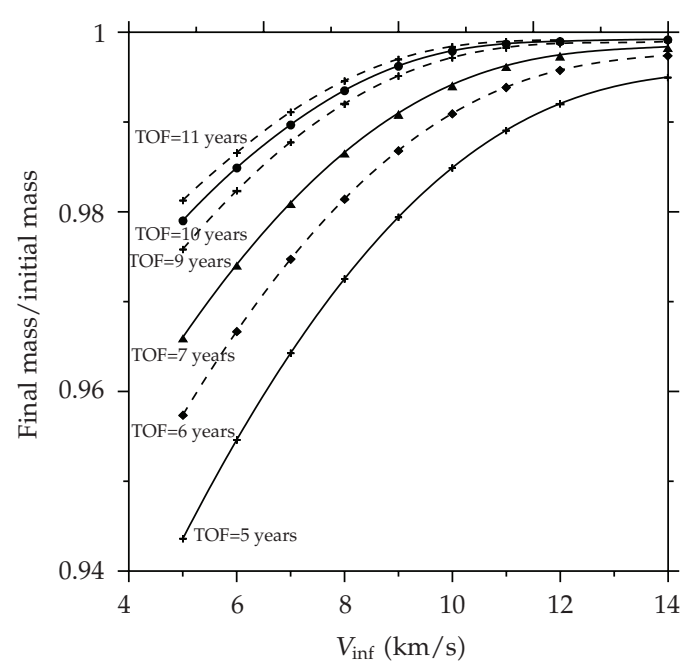

(b)

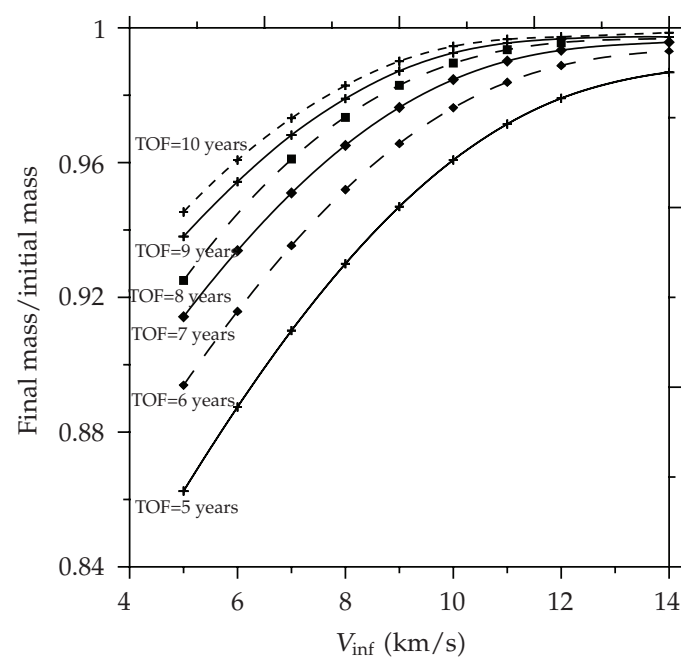

(d)

Figure 2: Curves for NEP as a function of the ratio $m_{f} / m_{0}$ for several TOFs. The effective specific powers are (a) $100 \mathrm{~W} / \mathrm{kg}$, (b) $80 \mathrm{~W} / \mathrm{kg}$, (c) $60 \mathrm{~W} / \mathrm{kg}$, (d) $30 \mathrm{~W} / \mathrm{kg}$.

Later, Smart-1 (ESA-2003) and Hayabusa (Japan-2003) were launched. With the successful demonstration made by the Deep Space 1, many studies have been performed to show the applicability and performance of Solar Electric Propulsion (SEP) for interplanetary missions (Brophy and Noca [1], Racca [7]). Previously, we did not consider this option for a trip to Saturn (Solórzano, Prado, and Sukhanov [8]).

It is known that the weakness of the SEP technology is the low levels of acceleration that it provides and in the reduced solar irradiance available for photovoltaic power generation at the outer reaches of the solar system. Nevertheless, these drawbacks can be avoided by a suitable design that allows the SEP system to operate efficiently for long periods using a wide range of input powers (Mengali and Quarta [9]). 


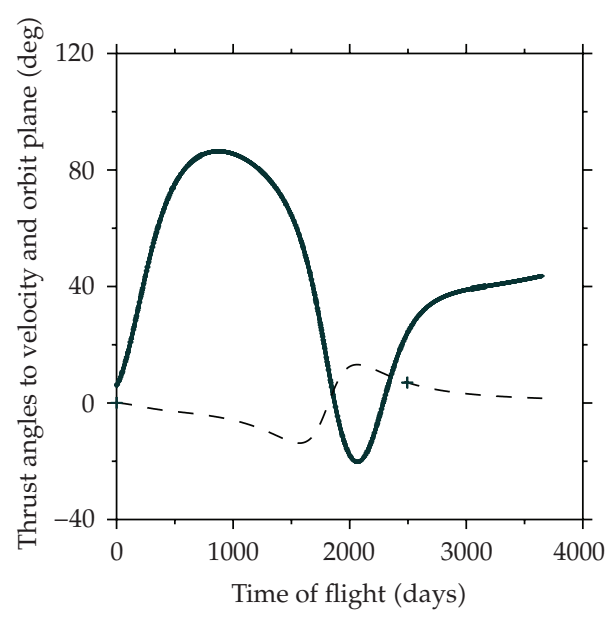

(a)

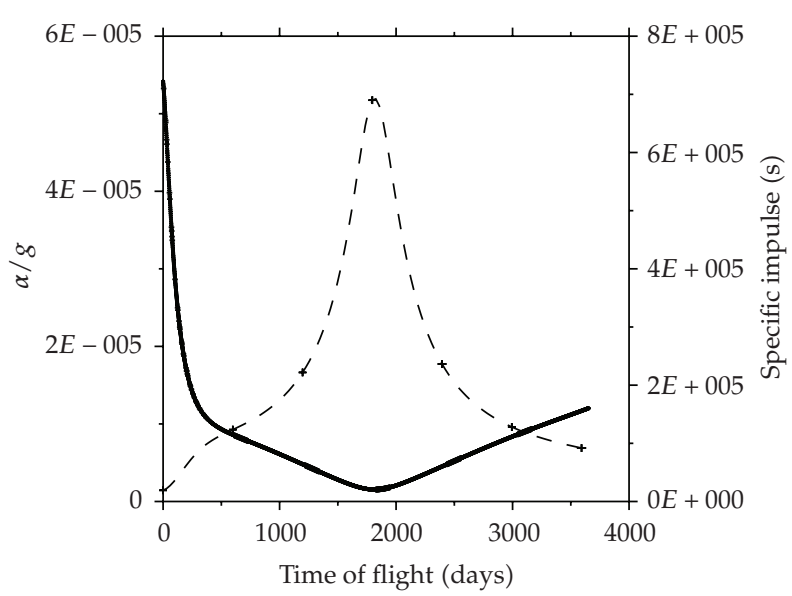

(b)

Figure 3: (a) Angle to the spacecraft velocity (continuous line) and angle to the orbital plane (discontinuous line). (b) Thrust vector (continuous line) and $\alpha / g$ and specific impulse (discontinuous line). Both for effective specific power of $50 \mathrm{~W} / \mathrm{kg}(\mathrm{NEP}), \mathrm{TOF}=10$ years and $V_{\mathrm{inf}}=5 \mathrm{~km} / \mathrm{s}$.

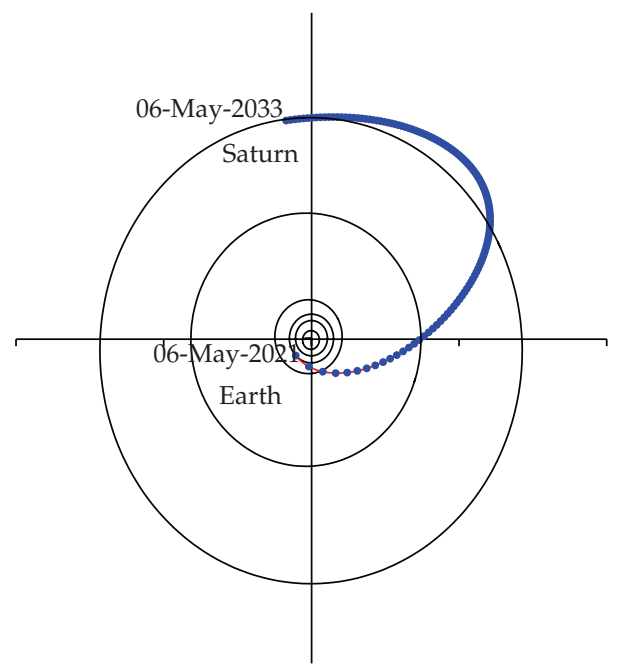

Figure 4: Trajectory of the flight Earth-Saturn with effective specific power $(\mathrm{REP})$ of $10 \mathrm{~W} / \mathrm{kg}$ and $V_{\text {inf }}=$ $10 \mathrm{~km} / \mathrm{s}$ on Earth's sphere influence (TOF = 12 years).

Here, we studied the possible advantages of SEP to reach Saturn. Figure 7 shows the trajectory of the flight Earth-Saturn projected on the ecliptic plane for the SEP.

Figure 8 shows the behavior of the relation final mass/initial mass versus $V_{\text {inf. }}$. It is visible that the relation of mass increases with the effective specific power. For example, when $V_{\text {inf }} \cong 0 \mathrm{~km} / \mathrm{s}$ and the specific initial power is $2 \mathrm{~W} / \mathrm{kg}$, the mass ratio is between 0.027 and 0.064 ( 5 years $\leq \mathrm{TOF} \leq 12$ years). For the case when $V_{\text {inf }} \cong 0 \mathrm{~km} / \mathrm{s}$ and specific initial power is $12 \mathrm{~W} / \mathrm{kg}$, the mass relation is between 0.33 and 0.73 ( 5 years $\leq \mathrm{TOF} \leq 12$ years).

The angle of the thrust with respect of the spacecraft velocity is shown in Figure 9(a) (continuous lines), however the angles of the thrust with the orbital plane take small values (discontinuous lines). 


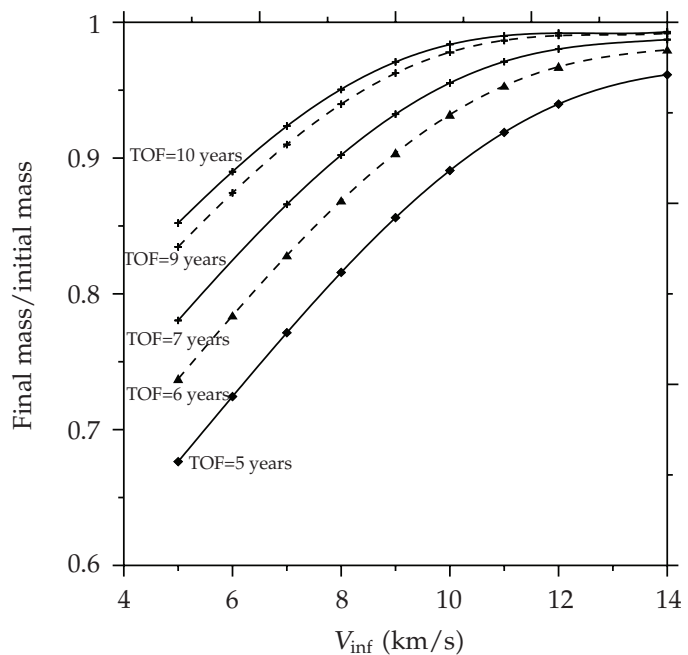

(a)

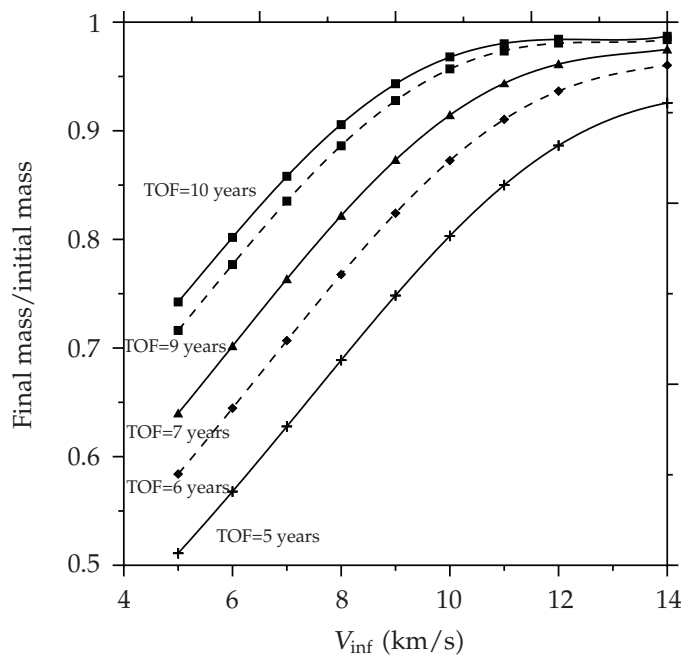

(c)

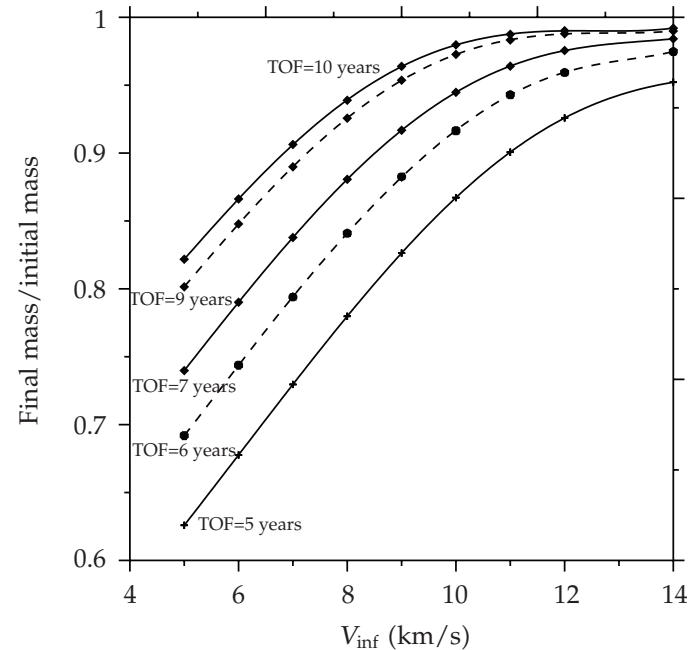

(b)

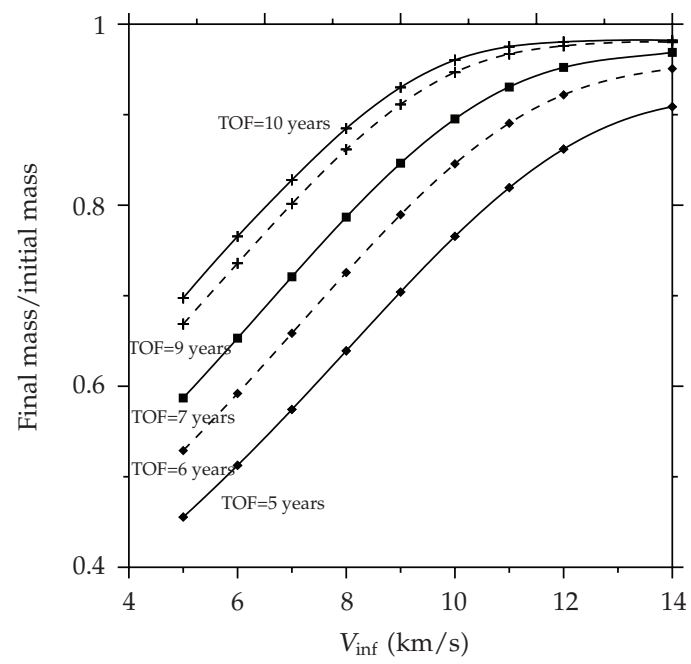

(d)

Figure 5: Curves for REP as a function of the ratio $m_{f} / m_{0}$ for several TOFs. The effective specific powers are (a) $10 \mathrm{~W} / \mathrm{kg}$, (b) $8 \mathrm{~W} / \mathrm{kg}$, (c) $6 \mathrm{~W} / \mathrm{kg}$, (d) $5 \mathrm{~W} / \mathrm{kg}$.

The behavior of the thrust is presented in Figure 9(b) (continuous lines). It is visible that the thrust has high values at the beginning of the transfers. The specific impulse (discontinuous lines) shows an opposite behavior. The maximum peak of the specific impulse happens when the thrust angle and the orbital plane suffer significant changes.

\section{Conclusions}

Low-thrust transfers of the limited power type were considered in this paper. The method of the transporting trajectory was used with the reference orbit composed by a set of short arcs of the keplerian orbits, while the transfer trajectory is subjected to low thrust. Since a maximum power provides a minimum propellant consumption, our goal was to maximize the $m_{f} / m_{0}$ 


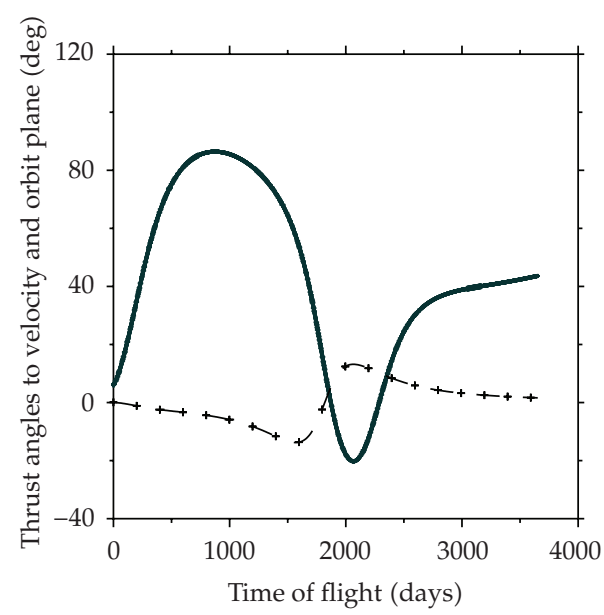

(a)

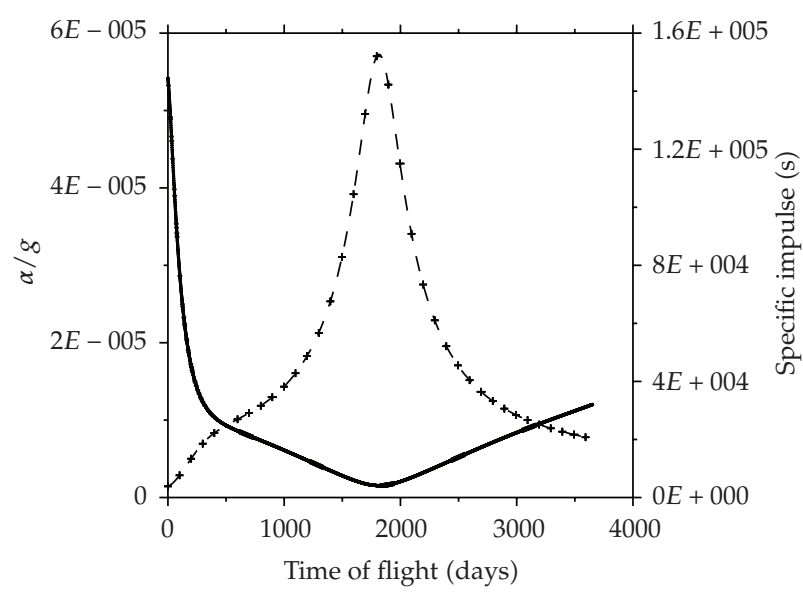

(b)

Figure 6: (a) Angle to the spacraft velocity (continuous line) and angle to the orbital plane (discontinuous line). (b) Thrust vector (continuous line) and $\alpha / g$ and specific impulse (discontinuous line). Both for effective specific power of $10 \mathrm{~W} / \mathrm{kg}(\mathrm{REP}), \mathrm{TOF}=10$ years, and $V_{\mathrm{inf}}=5 \mathrm{~km} / \mathrm{s}$.

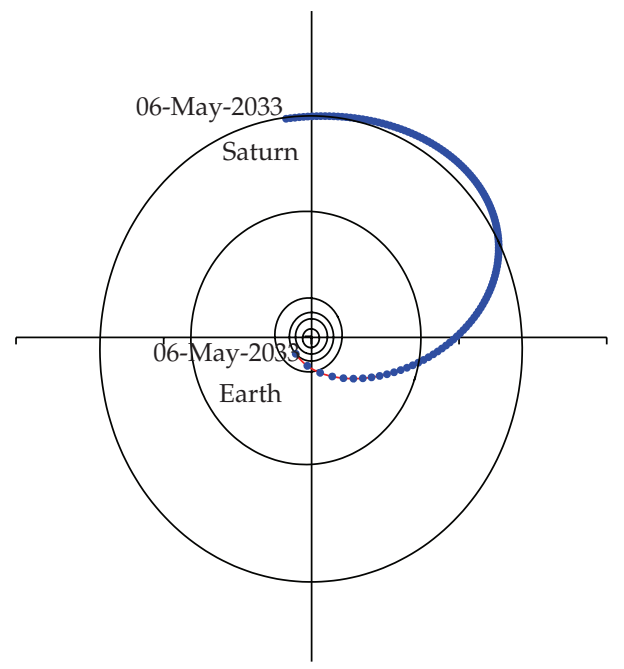

Figure 7: Trajectory for the flight Earth-Saturn with effective specific power (SEP) of $2 \mathrm{~W} / \mathrm{kg}$ and $V_{\text {inf }}=$ $10 \mathrm{~km} / \mathrm{s}$ on Earth's sphere influence (TOF = 12 years).

ratio. The NEP system permits to send more payloads, when compared to other options. If larger payloads were required, a nuclear reactor powered system would be needed. NEP is especially applicable for short trip time, large launch masses, and high-energy missions. The REP system appears to be more attractive for higher values of $V_{\text {inf }}$, due to the fact that, with low values for the specific power it is possible to send larger payloads, when compared to the NEP system. For other values of the $V_{\text {inf }}$, the REP delivers less science payload with proportional less power available for science instruments. The SEP systems can deliver large payloads for the case where the specific power, time of flight, and $V_{\text {inf }}$ are high. For the SEP systems a better option is a combination with gravity assisted maneuver. In general, maximum power provides a minimum propellant consumption. 


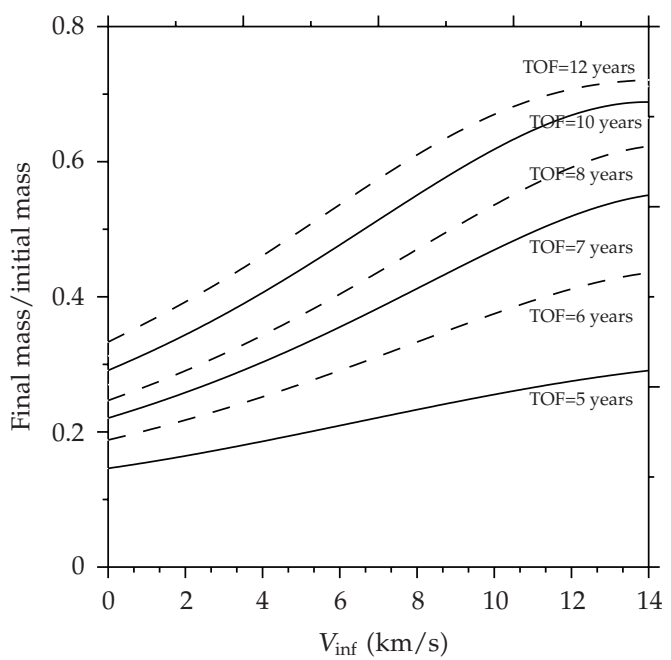

(a)

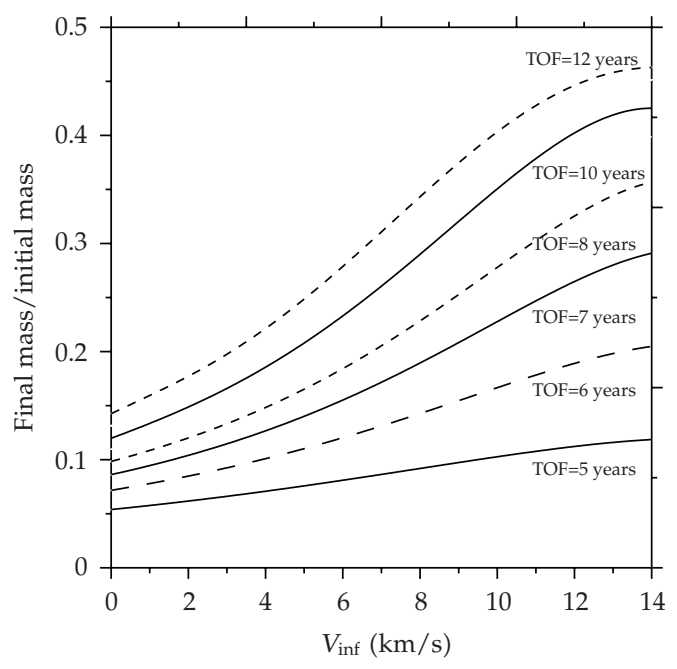

(c)

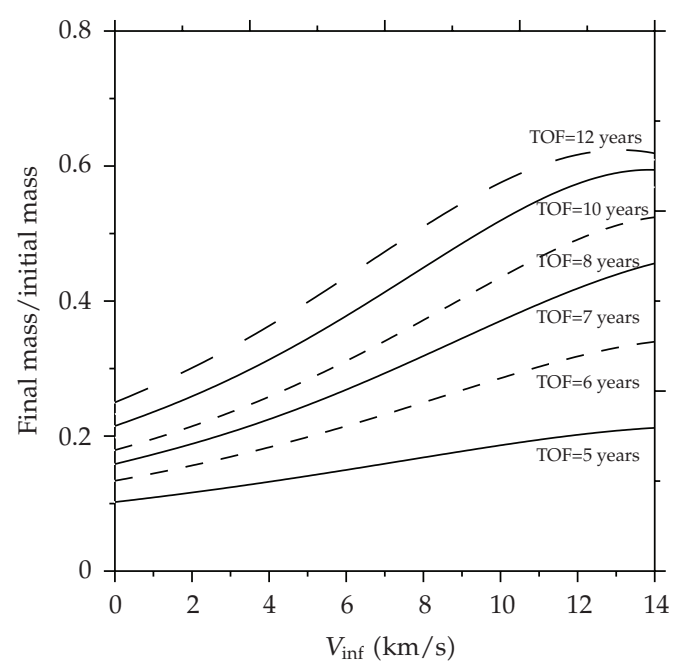

(b)

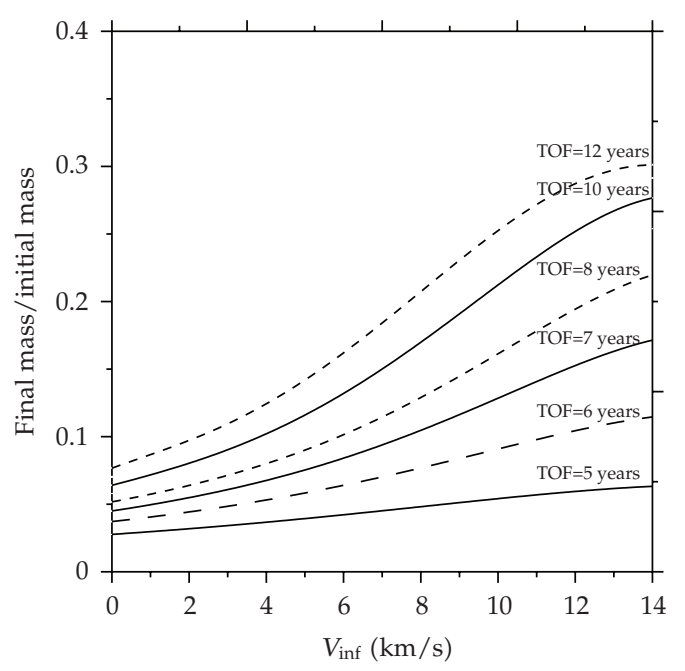

(d)

Figure 8: Curves for SEP as a function of the ratio $m_{f} / m_{0}$ for several TOFs. The effective specific powers are (a) $12 \mathrm{~W} / \mathrm{kg}$, (b) $8 \mathrm{~W} / \mathrm{kg}$, (c) $4 \mathrm{~W} / \mathrm{kg}$, (d) $2 \mathrm{~W} / \mathrm{kg}$.

\section{Nomenclature}

\section{Designate}

$$
\begin{array}{ll}
F_{T}=\dot{m}_{p} u & \text { thrust } \\
\mathrm{f}_{\mathrm{v}}=\mathrm{f}_{\mathrm{V}}(\mathrm{r}) & \text { gravatational acceleration } \\
I_{s p} & \text { specific impulse } \\
m=m(t) & \text { current spacecraft mass }\left(t_{0} \leq t \leq t_{1}\right) \\
m_{f} / m_{0} & \text { ratio between the final and initial mass } \\
m_{p}=m_{p}(t)=m_{0}-m & \text { propellet mass consumed by } t
\end{array}
$$




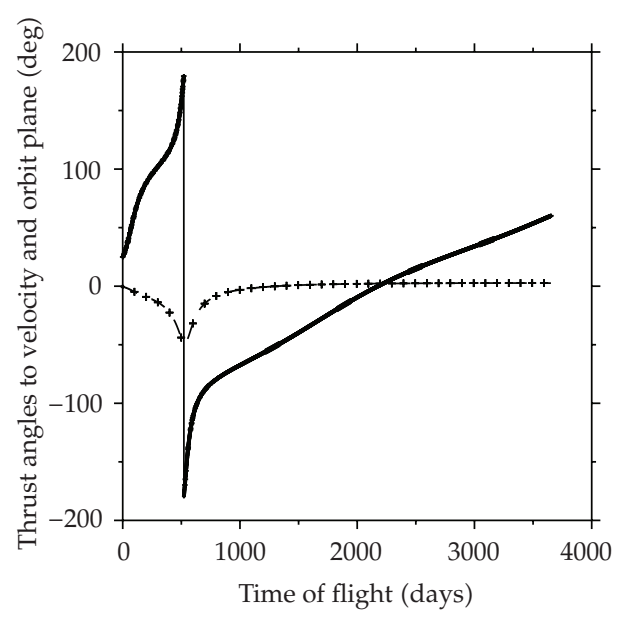

(a)

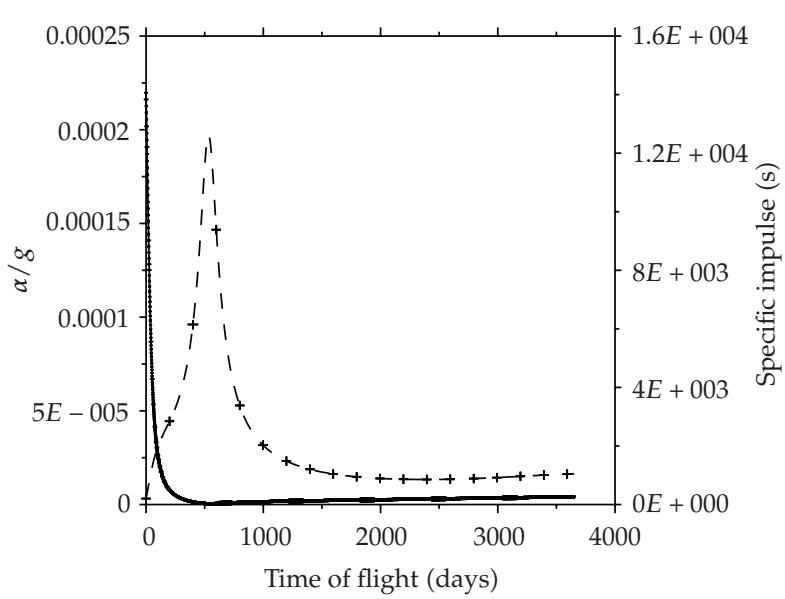

(b)

Figure 9: (a) Angle to the spacraft velocity (continuous line) and angle to the orbital plane (discontinuous line). (b) Thrust vector (continuous line) and $\alpha / g$ and specific impulse (discontinuous line). Both for effective specific power of $2 \mathrm{~W} / \mathrm{kg}(\mathrm{SEP}), \mathrm{TOF}=10$ years, and $V_{\text {inf }}=5 \mathrm{~km} / \mathrm{s}$.

$\begin{array}{ll}\dot{m}_{p}=\frac{d m_{p}}{d t}=-\dot{m} \geq 0 & \text { mass flow rate } \\ \vec{p}_{v} & \text { Lawden's primer vector } \\ t_{0}, t_{1} & \text { initial and final times } \\ u & \text { exhaust velocity } \\ V_{\text {inf }} & \text { velocity at infinity } \\ W & \text { electric power } \\ W_{e}=\eta W & \text { effective power } \\ \vec{\alpha}=\vec{\alpha}(t) & \text { acceleration vector } \\ \eta & \text { power efficiency (constant). }\end{array}$

\section{Acknowledgment}

The authors are grateful to the Foundation to Support Research in the São Paulo State, Brazil (FAPESP) for the research grant received under Contract 2008/10236-3 and 2007/04232-2.

\section{References}

[1] J. R. Brophy and M. Noca, "Electric propulsion for solar system exploration," Journal of Propulsion and Power, vol. 14, no. 5, pp. 700-707, 1998.

[2] J. P. Marec, Optimal Space Trajectories, Elsevier Science, Amsterdam, The Netherlands, 1979.

[3] V. Beletsky and V. Egorov, "Interplanetary flights with constant output engines," Cosmic Research, vol. 2, no. 3, pp. 303-330, 1964.

[4] A. A. Sukhanov, "Optimization of flights with low thrust," Cosmic Research, vol. 37, no. 2, pp. 182-191, 1999.

[5] A. A. Sukhanov, "Optimization of low-thrust interplanetary transfers," Cosmic Research, vol. 38, no. 6, pp. 584-587, 2000.

[6] M. D. Rayman and S. N. Williams, "Design of the first interplanetary solar electric propulsion mission," Journal of Spacecraft and Rockets, vol. 39, no. 4, pp. 589-595, 2002. 
[7] G. D. Racca, "Capability of solar electric propulsion for planetary missions," Planetary and Space Science, vol. 49, no. 14-15, pp. 1437-1444, 2001.

[8] C. R. H. Solórzano, A. F. B. Prado, and A. A. Sukhanov, "Analysis of electric propulsion system for exploration of Saturn," in Proceedings of the International Conference on Mathematical Problems in Engineering, Aerospace and Sciences (ICNPAA '08), Genoa, Italy, 2008.

[9] G. Mengali and A. A. Quarta, "Optimal trade studies of interplanetary electric propulsion missions," Acta Astronautica, vol. 62, no. 12, pp. 657-667, 2008. 


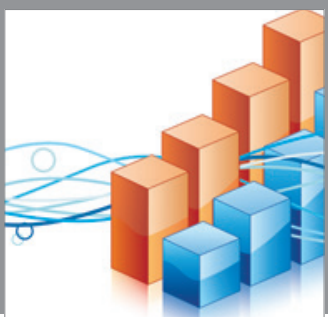

Advances in

Operations Research

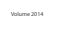

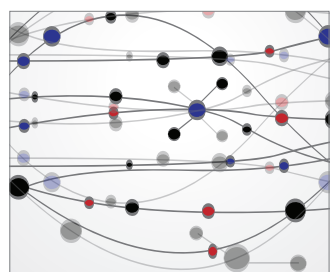

\section{The Scientific} World Journal
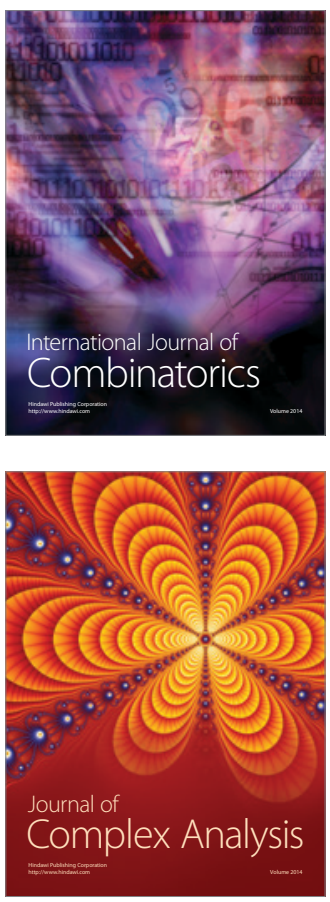

International Journal of

Mathematics and

Mathematical

Sciences
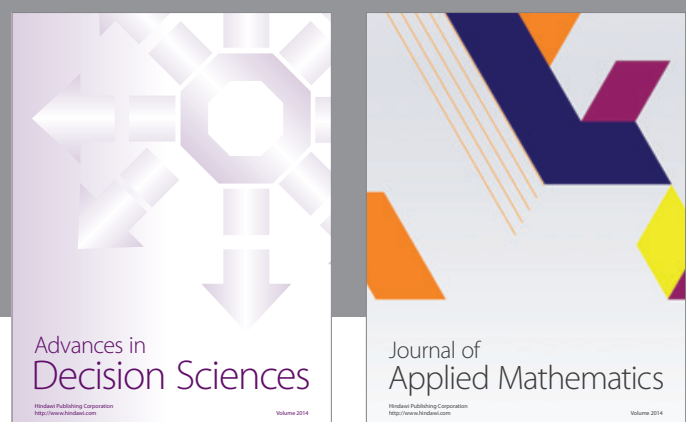

Journal of

Applied Mathematics
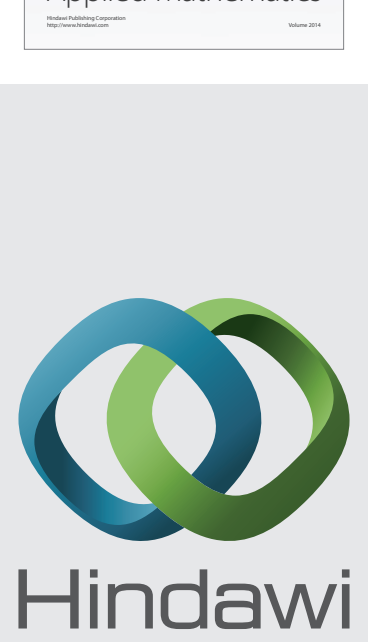

Submit your manuscripts at http://www.hindawi.com
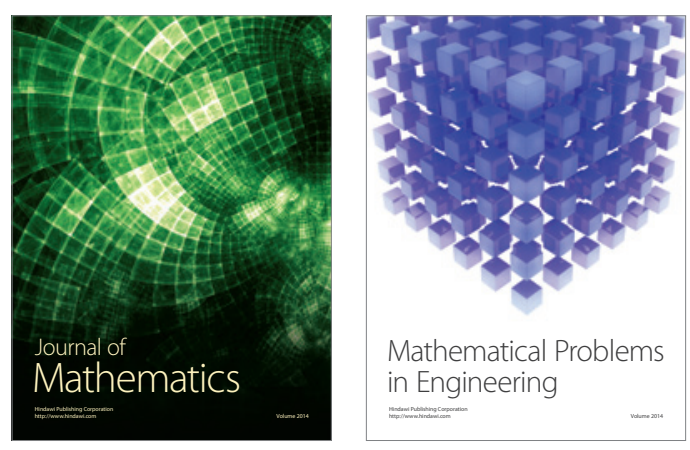

Mathematical Problems in Engineering
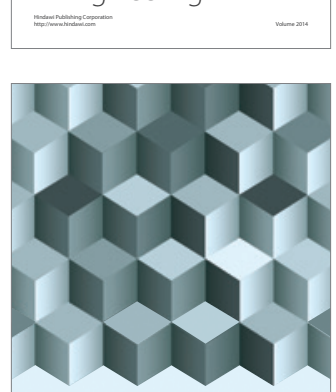

Journal of

Function Spaces
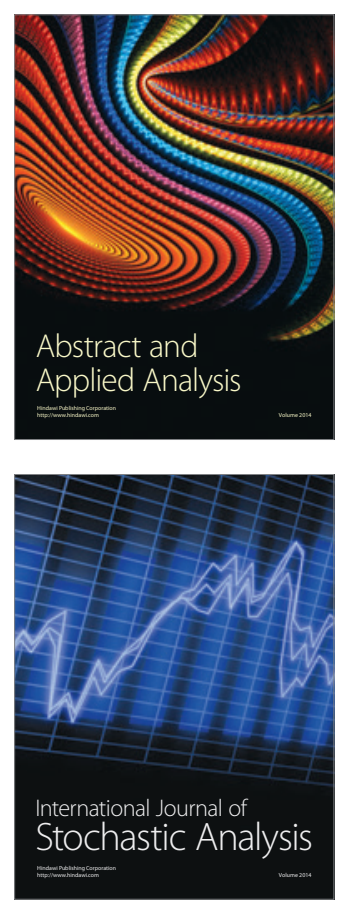

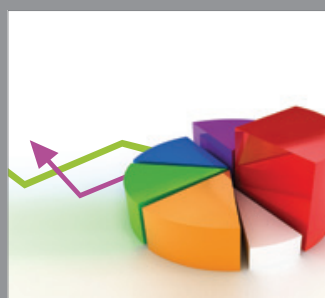

ournal of

Probability and Statistics

Promensencen
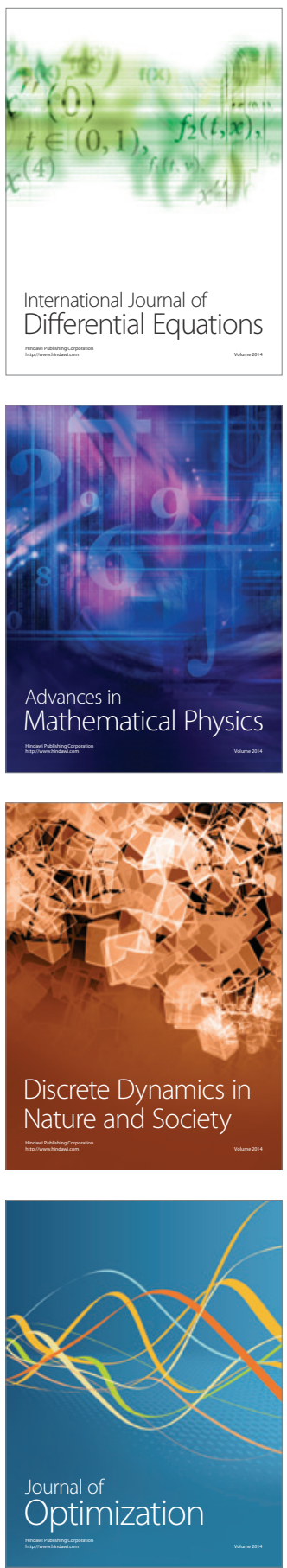